\title{
COMMENT
}

\section{Acute-on-chronic stress in the time of COVID-19: assessment considerations for vulnerable youth populations}

\author{
Mona Patel ${ }^{1}$ and Jean L. Raphael ${ }^{2}$ \\ Pediatric Research (2020) 88:827-828; https://doi.org/10.1038/s41390-020-01166-y
}

The global pandemic of the novel coronavirus disease 2019 (COVID-19) has highlighted struggles faced by marginalized populations worldwide. While pediatric patients are not among the highest physical health risk population affected by COVID-19, necessary public health measures have created adverse social emotional impacts on the health and well-being of children. ${ }^{1}$ With required public health measures such as closures of schools and community resource agencies including systems of care, children have become invisible through the pandemic, especially vulnerable youth who are dependent upon these services. ${ }^{2}$ In this issue, Gabrielli and Lund $^{3}$ reviewed chronic stress of youth living in poverty, youth with disabilities, and youth living in high conflict families and the overlay of acute stress related to the current pandemic on these populations. The authors also reviewed the concept of intersectionality of elevated rates of youth with disabilities among families living in poverty and an increased likelihood of experiencing family conflict. In conclusion, Gabrielli and Lund recommend that pediatricians and family partner to recognize and address the impacts of acute stress of the current pandemic, particularly for vulnerable populations with communication and coordination of support systems.

In this commentary, we focus on the impact the pandemic has had in highlighting disparities related to sociodemographic and environmental factors for children and their families. Highly populated counties with poor air quality, multigenerational households in predominantly Black and Latino neighborhoods lead to increase community spread of COVID-19 with an increased risk of morbidity and mortality from COVID- $19^{4}$ due to inability to enact physical distancing restrictions. Children with disabilities have a unique challenge during the current pandemic, as isolating measures such as school closures lead to creation of protective home environments that have been shown to decrease risk of contraction of COVID-19, unless living in crowded living environments. ${ }^{5}$ With initial limitation of health care access and concerns around safe delivery of medications and durable medical equipment, pediatric providers were most concerned with medically complex populations of children. With evidence that family stress is increased while caring for medically fragile children with overlying acute stress of the pandemic leading to limited access to home health care and in-home supportive services, this further leads to a challenging home environment. The nature of stay-at-home orders has increased social isolation, increased economic and health vulnerabilities of victims of intimate partner violence, which had recently seen a decline in reported rates prior to COVID-19. ${ }^{6}$ Impacts of families isolating together in strenuous partner relationships lead to increased stress on children when they experience continued trauma from dysfunctional family relationships. Furthermore, access to resources for mental health and community partner services has been affected during COVID19 for families with high conflict. Most pertinent for children living in these household conditions is a relative "invisibility" to this issue as children are facing limited interaction with others outside of the family during current times.

As Gabrielli and Lund highlight, some of the most devastating consequences of the pandemic on children includes the lack of opportunity for socialization and peer to peer engagement, which is enriched in school and community settings. In addition, schools and community-based agencies serve as resources for safety net resources, such as food, behavioral and mental health collaborative environments, and for housing and financial supports. Furthermore, access to such safety net resources with closures and diminished staff and the effect of familial financial instability with job loss can further create increased stress on families. ${ }^{7}$ With reduced in-person access to safety net resources including education and supportive services, many agencies are converted to virtual platforms to deliver such access. Unfortunately, consideration needs to be placed on the digital divide, which disproportionately affects racial and ethnic minorities, persons with disabilities, rural populations, and families with a lower socioeconomic status. ${ }^{8}$ The impacts of the COVID-19 global pandemic has unfortunately highlighted the socioeconomic, environmental, and racial/ethnic disparities our children and families have faced for centuries.

In consideration of the acute stress caused by the COVID-19 pandemic on an already chronically stressed, vulnerable, marginalized population, access to quality behavioral and mental health services is critical. Honest and clear communication with children in all populations related to the uncertainty of the pandemic without overwhelming with fear is key. ${ }^{9}$ An acknowledgement of stress contagion, ${ }^{10}$ the direct and indirect effects of stress related to the pandemic on families compound existing chronic stress, is important, particularly for vulnerable populations. With uncertainty and fear of the virus, along with social isolation, and potentially increased job loss with worsening food and housing instability, stress contagion adds to the impacts of mental health wellness for families.

Historically, schools play an important role in access to mental health services, with greater than one-third of adolescent populations seeking services in such settings. In addition, adolescents with public insurance, low-income households, and from racial/ethnic minority groups almost exclusively use schoolbased mental health resources. With closures during the

${ }^{1}$ Department of Pediatrics, Children's Hospital Los Angeles, Los Angeles, CA, USA and ${ }^{2}$ Center for Child Health Policy and Advocacy, Baylor College of Medicine, Houston, TX, USA Correspondence: Mona Patel (mpatel@chla.usc.edu)

Received: 16 August 2020 Accepted: 20 August 2020

Published online: 17 September 2020 
pandemic, access is limited. ${ }^{11}$ Continued policy efforts for the expansion of health insurance coverage for mental health services with increased integration of high-quality mental health screening and therapeutic services into pediatric primary care is important. ${ }^{12}$ Supportive screening for adverse childhood experiences (ACEs) is critical, given a disproportionate effect on Black non-Hispanic and Latino populations with now rising rates of poverty and continued social isolation due to current public health measures. ${ }^{13,14} \mathrm{~A}$ recent publication reviewed worldwide impacts of COVID-19 on operations of non-governmental organizations in 43 countries serving $>450,000$ vulnerable families reveals devastating early effects of the pandemic on their provision of services. ${ }^{15}$ There is an intercurrent need to innovate and revise previous strategies on providing services to vulnerable children and their families since many agencies are unable to serve with current restrictions in place. In addition, adaptability in virtual and remote options and facilitating connections are critical in linking families to much needed services. Finally, empowering local communities to be able to connect and build frameworks to help vulnerable families is the key for creating sustainable long-term support systems.

The Coronavirus Aid, Relief, and Economic Security (CARES) Act passed through bipartisan effort, and signed into law on 3/27/20, sought to help enhance unemployment benefits. In addition, it created health access provisions including unprecedented leniency in virtual care access offerings across all payer platforms and funding for pediatric healthcare providers who had suffered from low-volume care under crippling safer-at-home initiatives. While advocacy for such federal level support is helpful, continued public policy allowing continued supports for families who are further marginalized during this pandemic will be critical to limiting long-term consequences of this virus on the socioemotional well-being and health outcomes of our children. Expansion of supports for financial stability for families living in poverty, improvement of access to digital health for vulnerable populations to aid in innovative resource management for systems of care, and promotion of supportive access to behavioral health services with effective screening for ACEs will improve child health outcomes in spite of the current pandemic.

\section{AUTHOR CONTRIBUTIONS}

Both authors have made substantial contributions to conception and design, drafting the article, or revising it critically for important intellectual content and final approval of the version to be published.

\section{ADDITIONAL INFORMATION}

Competing interests: The authors declare no competing interests.

Publisher's note Springer Nature remains neutral with regard to jurisdictional claims in published maps and institutional affiliations.

\section{REFERENCES}

1. Wong, C. A., Ming, D., Maslow, G. \& Gifford, E. J. Mitigating the impacts of the COVID-19 pandemic response on at-risk children. Pediatrics 146, e20200973 (2020).

2. Naseri, A. \& Hosseini, M. S. Do not neglect the children: considerations for COVID19 pandemic. Indian Pediatr. 57, 583-584 (2020).

3. Gabrielli, J. \& Lund, E. Acute-on-chronic stress in the time of COVID-19: assessment considerations for vulnerable youth populations. Pediatr. Res. https://doi. org/10.1038/s41390-020-1039-7 (2020).

4. Correa-Agudelo, E. et al. Identification of vulnerable populations and areas at higher risk of COVID-19 related mortality in the U.S. Health Sciences. https://doi. org/10.1101/2020.07.11.20151563 (2020).

5. Sholas, M. G. The actual and potential impact of the novel 2019 coronavirus on pediatric rehabilitation: a commentary and review of its effects and potential disparate influence on Black, Latinx and Native American marginalized populations in the United States. J. Pediatr. Rehabil. Med. https://doi.org/10.3233/PRM200722 (2020).

6. Kaukinen, C. When stay-at-home orders leave victims unsafe at home: exploring the risk and consequences of intimate partner violence during the COVID-19 pandemic. Am. J. Crim. Just. 45, 668-679 (2020).

7. Silliman Cohen, R. I. \& Bosk, E. A. Vulnerable youth and the COVID-19 pandemic. Pediatrics 146, e20201306 (2020).

8. Chang, B. L. et al. Bridging the digital divide: reaching vulnerable populations. J. Am. Med. Inf. Assoc. 11, 448-457 (2004).

9. Dalton, L., Rapa, E. \& Stein, A. Protecting the psychological health of children through effective communication about COVID-19. Lancet Child Adolesc. Health 4, 346-347 (2020).

10. Liu, C. H. \& Doan, S. N. Psychosocial stress contagion in children and families during the COVID-19 pandemic. Clin. Pediatr. 59, 853-855 (2020).

11. Ali, M. M. et al. Utilization of mental health services in educational setting by adolescents in the United States. J. Sch. Health 89, 393-401 (2019).

12. Moreno, C. et al. How mental health care should change as a consequence of the COVID-19 pandemic. Lancet Psychiatry 7, 813-824 (2020).

13. Saleem, $\mathrm{H}$. et al. The rise of adverse childhood experiences during the COVID-19 pandemic. Psychol. Trauma 12, S193-S194 (2020).

14. K, R., Petkova, E., Bhui, K. S. \& Schulze, T. G. A global needs assessment in times of a global crisis: world psychiatry response to the COVID-19 pandemic. BJPsych Open 6, e48 (2020).

15. Wilke, N. G., Howard, A. H. \& Pop, D. Data-informed recommendations for services providers working with vulnerable children and families during the COVID-19 pandemic. Child Abuse Negl. https://doi.org/10.1016/j.chiabu.2020.104642 (2020). 